\title{
Distance Measurement for Sensorless 3D US
}

\author{
Peter Hassenpflug, Richard Prager, Graham Treece, and Andrew Gee \\ Department of Engineering, University of Cambridge, Trumpington Street, \\ Cambridge, UK, CB2 1PZ, ph305@cam.ac.uk
}

\begin{abstract}
Previously it was thought that speckle-based distance measurement techniques worked best over very small distances. We show that this is not necessarily the case. This result has significance for the design of accurate sensorless freehand 3D ultrasound systems that are convenient to use in a clinical setting.
\end{abstract}

\section{Introduction}

Freehand 3D ultrasound, in which B-scan slices and probe trajectory are simultaneously recorded, provides a valuable clinical tool for volume measurement and the analysis of complex geometry. However, the need for a position sensor with a fixed transmitter or cameras external to the ultrasound probe is inconvenient in a clinical environment. The goal is thus to use less intrusive techniques for probe tracking without compromising the spatial accuracy of the overall system.

In this paper, we are concerned with estimating the probe motion perpendicular to the plane of the 2D B-scan (in the elevational direction). Speckle decorrelation [1] and speckle regression [2] can both be used to measure the distance between neighbouring B-scan slices in this direction provided that the spacing of the slices is less than the width of the ultrasound beam. In our previous work we regarded this as the only restriction on the usefulness of these algorithms. In this paper we show that this is not the whole story and there is also a lower limit to the useful working range of the speckle-based algorithms.

\section{Distance Measurement}

Speckle-based distance measurement is based on the fact that there is a roughly Gaussian relationship between the correlation (or regression gradient) of the echo envelope intensities of a pair of patches of speckle, and their distance apart. The precise shape of this relationship depends on the beam profile, but it is usually of the form shown in the top left graph in figure 1. For very small distances, the shallow gradient of the curve means that small errors in either the curve shape, or the measured correlation coefficient, result in large percentage errors in the predicted distance.

A speckle phantom was scanned with a 5-10 MHz linear array probe. The radio frequency data was recorded at $66.67 \mathrm{MHz}$ for sixty parallel slices $0.02 \mathrm{~mm}$ apart. Models were built of the elevational decorrelation rate for 136 patches of about 2500 intensity samples spread across the slice. Distance measurements 

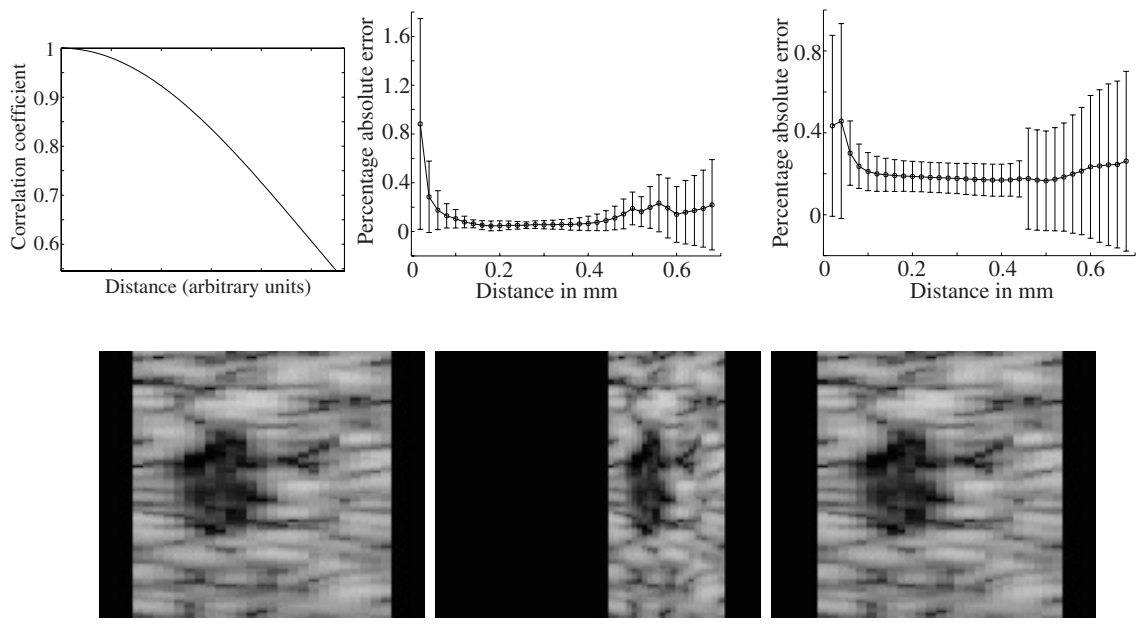

Fig. 1. Top left: a typical speckle decorrelation curve. Top mid \& right: percentage errors for training and test data vs. size of distance measured. Bottom left: correct reslice of $2 \mathrm{~mm}$ diameter void. Bottom mid \& right: reslices based on decorrelation measurements over $0.04 \mathrm{~mm}$ and $0.16 \mathrm{~mm}$ respectively.

were made over various ranges from $0.02 \mathrm{~mm}$ up to $0.68 \mathrm{~mm}$ using one patch with beam width $\approx 1 \mathrm{~mm}$. The mean and standard deviation of the absolute errors are shown in the top middle graph for the data that was used to build the model, and in the top right graph for different data of a similar phantom.

The bottom left figure shows an accurate reslice through a $2 \mathrm{~mm}$ diameter anechoic void in the phantom. The horizontal spacing of the slices is $0.16 \mathrm{~mm}$. The bottom right figure shows the same reslice with positions calculated using decorrelation between all the patches on the slices. The spacing is similar to the correct answer. The bottom middle reslice uses positions derived from the sum of four decorrelation calculations between slices $0.04 \mathrm{~mm}$ apart, by using three extra slices between each of those shown. Here, the horizontal spacing is poor.

\section{Conclusions}

The accuracy of speckle-based distance measurement techniques degrades for distances less than about $10 \%$ of the beam width.

\section{References}

1. J-F. Chen, J. B. Fowlkes, P. L. Carson, and J. M. Rubin. Determination of scanplane motion using speckle decorrelation: theoretical considerations and initial test. International Journal of Imaging Systems Technology, 8:38-44, 1997.

2. R. W. Prager, A. H. Gee, G. M. Treece, C. J. C. Cash, and L. H. Berman. Sensorless freehand $3 \mathrm{D}$ ultrasound using regression of the echo intensity. Ultrasound in Medicine and Biology, 29(3):437-446, March 2003. 